einem kurzen Text, der komplett auf eine Info-Stelenseite passt, kann die Animation auch abgeschaltet werden. Dies bewirkt eine angenehmere und ruhigere Darstellung. Die genannten Einstellungen bilden den Rahmen für eine Info-Stele, in den nun beliebig viele Seiten hinzugefügt werden können.

Wir haben uns - pro Bildschirmorientierung - für ein festes Layout entschieden, um die Arbeit für die "Redakteure" möglichst einfach und effizient zu halten. Es stehen die Inhaltselemente Titel, Untertitel, Bild bzw. Video und der beschreibende Text pro Seite zur Verfügung. Soll eine Seite eine abweichende Verweildauer erhalten, so kann diese hier ebenso festgelegt werden, wie Überblendeffekte und ggf. besondere Farbgestaltungen. Als Layoutvariante kann das Bild auch für den gesamten Hintergrund verwendet werden. Für manche Inhalte (z.B. Baustellen-Webcam) entstehen auf diese Weise interessantere Präsentationen. Über die Zeitsteuerung kann jeder Seite ein Sichtbarkeitszeitraum zugeordnet werden. Bei Termin gebundenen Inhalten entfällt dadurch der zeitnahe manuelle Eingriff und entsprechende Seiten können komfortabler vorbereitet werden.

In der Verwaltungsoberfläche steht eine Vorschaufunktion zur Verfügung, um Layout und Inhalte prüfen zu können. Da am Arbeitsplatz der Bearbeiter oftmals Bildschirme verwendet werden, deren Auflösung sich von der Info-Stelenhardware unterscheidet, ist dies ein sehr praktisches Feature.

In der Württembergischen Landesbibliothek besteht - u.a. auch durch die verschiedenen Baumaßnahmen - ein verstärkter Informationsbedarf unseren Nutzern gegenüber. Daher sollen im Bestandsgebäude in nächster Zeit noch weitere Info-Terminals aufgestellt werden.

Stephan Abele

\title{
Kassenautomaten mit verbessertem Quittungsdruck
}

Zur Bezahlung der verschiedenen Bibliotheksgebühren (Jahresgebühr, Portoersatz, Mahnungen usw.) stehen in der Württembergischen Landesbibliothek für unsere Benutzer bereits seit vielen Jahren zwei Kassenautomaten der Firma HESS zur Verfügung. Die Bezahlung ist sowohl mit Bargeld als auch mit der Girokarte möglich.

Die Kommunikation zwischen dem Bibliothekssystem aDIS/BMS und dem Kassenautomaten erfolgt mit dem NCIP-Protokoll. In der bisher eingesetzten Version wurde nur der jeweilige Saldo des Benutzerkontos zwischen Kassenautomat und Bibliothekssystem ausgetauscht. Der Nachteil dieser Lösung war, dass für den Benutzer kein detaillierter Quittungsdruck erstellt werden konnte. Wurde eine ausführliche Quittung benötigt, musste die Bezahlung an der Barkasse in der Leihstelle erfolgen. Im Zuge des aDIS/BMS-Update-Projektes wurde im Jahr 2015 die Erweiterung der NCIP-Schnittstelle insbesondere für Gebühreneinzelauflistung - durch die Firma aStec beauftragt. Auf der anderen Seite musste auch die Automatensoftware erweitert werden, so dass die umfangreicheren Daten, die aDIS ausliefert, verarbeitet werden können. Diese Software konnte im November 2015 fertiggestellt werden. Am 28.01.2016 wurde nun auf den Kassenautomaten diese neue Softwareversion installiert und auf aDIS/BMS-Seite die entsprechenden Erweiterungen aktiviert.

Der Benutzerdialog hat sich aufgrund der erweiterten Funktionalität etwas geändert.

Auf dem Bildschirm werden alle Gebührenposten dargestellt. Die primäre Sortierung erfolgt nach der Gebührenart (Nutzungsgebühr, Fernleihgebühr, usw.). Innerhalb der Gebührenart wird chronologisch sortiert. Zunächst sind alle Posten als „zu zahlen" aktiviert. Auf dem Touchbildschirm wird nun eine "Zahlen?"-Spalte angezeigt. Der Benutzer kann per Touchbedienung einzelne Posten von der Zahlung ausnehmen. Somit können beispielsweise 
nicht sperrrelevante Fernleihgebühren zunächst für die Zahlung unberücksichtigt bleiben. Ausnahme:

Benutzungs- und Ersatzausweisgebühren müssen stets bezahlt werden.

Sowohl im Bildschirmdialog, als auch auf dem

Quittungsdruck werden alle bezahlten Posten mit ausführlicher Beschreibung und ggf. mit Kurztitel ausgegeben.

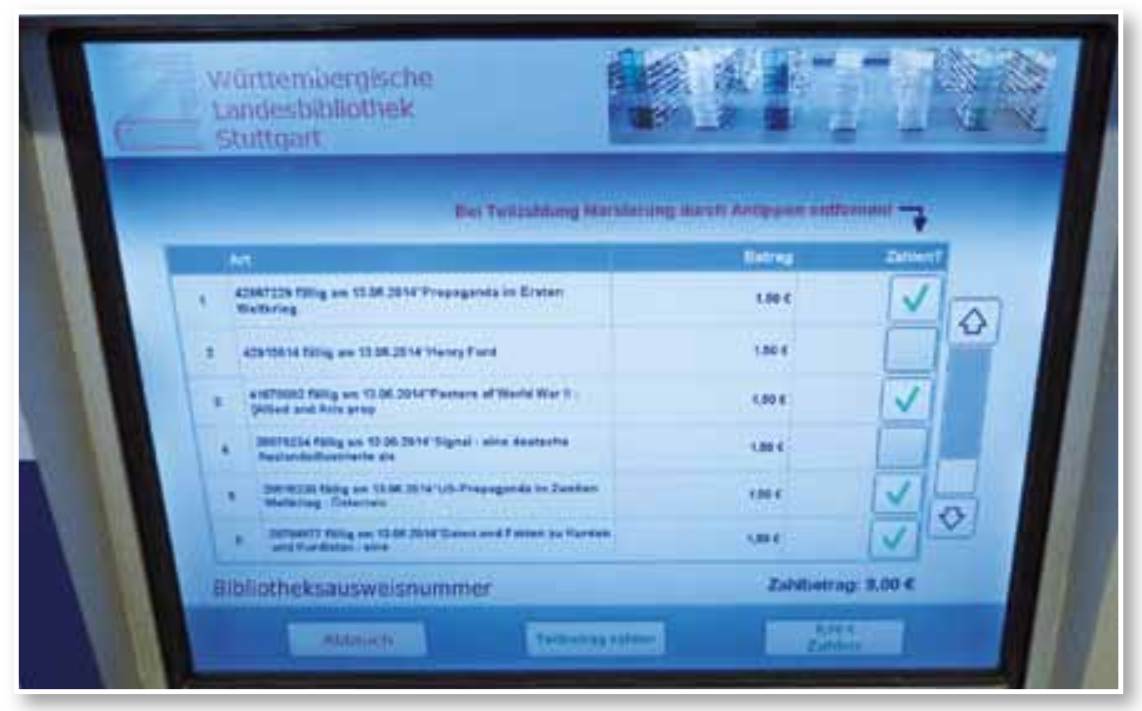

Erweiterte Bildschirmdarstellung

Durch die verbesserten Zahlungsmöglichkeiten am Kassenautomaten kann zukünftig die Barkasse abgeschafft werden.

Einer der beiden Automaten arbeitete intern noch mit ISDN-Technik. Da für die nächste Zeit der ISDN-Netzanschluss gekündigt ist, wurden im Zuge dieser Update-Arbeiten gleichzeitig alle ISDNAbhängigkeiten beseitigt und die Außenanbindung zum Zahlungsdienstleister auf allgemeine Netzwerktechniken umgestellt.

\section{Stephan Abele}

Gebühren direkt am Kassenautomaten kassieren.

\section{Mehr Service und Sicher- heit für Ihre Bibliothek.}

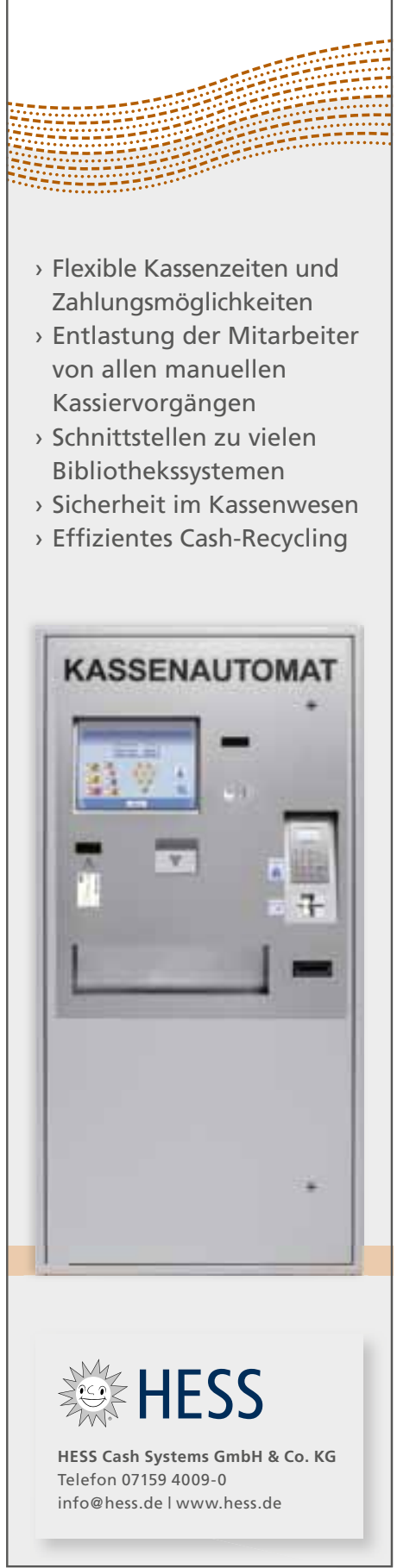

